\title{
Defining Virtual Humanoid Robotics with Modeling
}

\section{Shaikh $\mathrm{MS}^{* *}$ and Shaikh $\mathbf{R}^{2}$}

${ }^{1}$ KYDSC Trust's, Institute of Management and Science (IMS), North Maharashtra University, Bhusawal, M.S, India

${ }^{2}$ Department of Computers, G. H. Raisoni Institute of Engineering and Management, Jalgaon, M.S, India

\begin{abstract}
We have no doubt to state Virtual Humanoid Robots (VHRs) are the ultimate level of Artificial Intelligence which change the scenario of world and human technologies, it would be applicable in all domains of technology with common factor Ultra Artificial Intelligence (UAI) with disappear and appear ability by any means which boost to our civilization from Type-0 to type-1 civilization at least and would be first step to compete with Aliens technology, if exist (hypothesis only). We would like to define term Virtual Humanoid Robotics (VHR) as "it's Humanoid Robotics with UAl and has ability to transform from Physical to Virtual by any Internal (Humanoid Self-Control) or External (HumanControl) mode activation mechanism". VHR is future technology which will use energy from Sun (or Space), Internet of Things (IOT) with RFID USN, Bigdata and Self-learning and healing mechanism. Now we would like to generate future utopia front of your eyes with initial modeling to coined term VHR in this short communication.
\end{abstract}

Keywords: Humanoid robotics; Bionic brain; UAI; Virtual humanoid robotics; Robotics teleportation

\section{Modeling to VHR}

\section{VHR-basic engineering model}

We depicted in our first model "VHR-Basic Engineering" that there we need to extent our fundamental engineering aspect of Humanoid to Virtual Humanoid robotics domain [1,2]. Hence model divided into two broad chambers as Humanoid Chamber and to give virtual ability Virtualization chamber. As we can analyze from model for successful humanoid building we need advanced Humanoid robotics hardware's which link to Bionic Brain as similar to human brain mimic in the form of UAI which further cascade to Advanced Humanoid Operating System and Communication Interfaces [3]. After successful engineering of first segment successful physical humanoid can build but to next level i.e. to convert physical humanoid into virtual and back from virtual to physical we doesn't need to modify hardware but to strongly need to give extension to existed. Hence Virtualization chamber exhibits this regard in model (Figure 1). The virtualization chamber has two functional blocks to engineer Advanced Physical to Virtual Mode transfer Units and Light/Projection/Optical/Teleport interfaces Engineering [4].

\section{Physical-to-Virtual modes switch model}

Our second purposed model "Physical-to-Virtual Mode Switch" model one of the essential VHR engineering models, in another word can say expansion and detail discussion on Virtualization Chamber

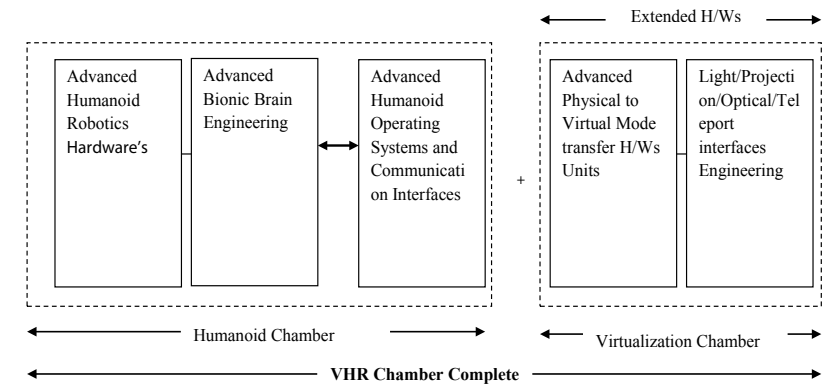

Figure 1: VHR-basic engineering model second part of our first model. Its lucid and clear representation of concept in model diagram we considered three different possible modes viz. M1, M2 and M3 which may be increase in future with technological advancement and new methods of virtualization [5]. The mode M1 has highest priority to implement VHR where Humanoid hardware itself has ability to appear and disappear itself with self-control (Internal Control) which is only hypothesis right now [6-8]. The second mode M2 has possible and second priority Teleportation and lot research going on this Mode M2 by several premium university and institutions scholars (Figure 2). The last mode M3 is easiest one but not satisfactory where virtualization engineer using virtual and augmented reality [8].

\section{Conclusion}

We have discussed two models and with the help of them try to

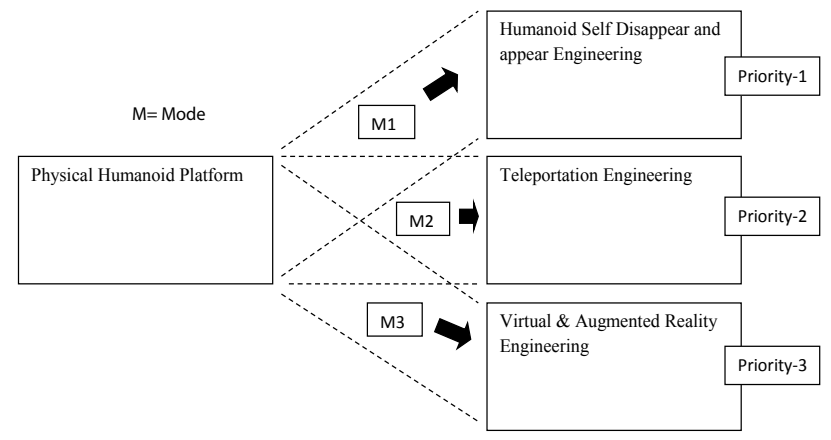

Source: Prof. Md. Sadique Shaikh

Figure 2: Physical-to-Virtual modes switch model.

*Corresponding author: Md. Sadique Shaikh, KYDSC Trust's, Institute of Management and Science (IMS), North Maharashtra University, India, Tel: 0257 226 4881; E-mail: sids_nsk@rediffmail.com

Received September 24, 2018; Accepted October 24, 2018; Published October 29, 2018

Citation: Shaikh MS, Shaikh R (2018) Defining Virtual Humanoid Robotics with Modeling. Adv Robot Autom 7: 189. doi: 10.4172/2168-9695.1000189

Copyright: (c) 2018 Shaikh MS, et al. This is an open-access article distributed under the terms of the Creative Commons Attribution License, which permits unrestricted use, distribution, and reproduction in any medium, provided the original author and source are credited. 
learn one of the promising and world change future technology Virtual Humanoid Robotics where Humanoid not only seems to be like human in near future but also will have ability to Avatar itself. This would be very helpful to send humanoid virtually in deep-space, on stars and planets to understand universe closely with teleportation or internal humanoid mechanism. VHR also ultimate level of AI hence might be shift mankind race on planet earth from Type-0 civilization to Type-1 civilization as shown in sci-fi movies.

\section{References}

1. Shaikh MS (2013) Analysis and modeling of Strong A.I to engineer BIONIC brain for humanoid robotics application. American Journal of Embedded System and Applications 1: 27-36.

2. Shaikh MS (2017) Ultra Artificial Intelligence (UAI): Redefing Al fir New Research Dimension. Adv Robot Autom 6: 1-3.
3. Shaikh MS (2017) Fundamental Engineering for Brain-Computer Interfacing $(\mathrm{BCl})$ : Initiative for Neuron-Command Operating Devices. Computational Biology and Bioinformatics 5: 50-56.

4. Shaikh MS (2018) Defining ultra-artificial intelligence (UAI) implementation using bionic (biological-like-electronics) brain engineering insight. MOJ App Bio Biomech 2:127-128.

5. Shaikh MS (2018) Artificial Intelligence Engineering for Cyborg Technology Implementation. Robot Autom Eng J 3: 1-3.

6. Shaikh MS (2018) Engineering Insight for Humanoid Robotics Emotions and Violence. Robot Autom Eng J 3: 1-8.

7. Shaikh MS (2018) Defining Cyborg Intelligence for Medical and Super-Human Domains. Trends in Technical \& Scientific Research 2: 1-2.

8. Shaikh MS (2018) Ultra artificial intelligence (UAI) engineering for robotics violence control, detect and corrective measures. Int Rob Auto J 4: 242-243. 